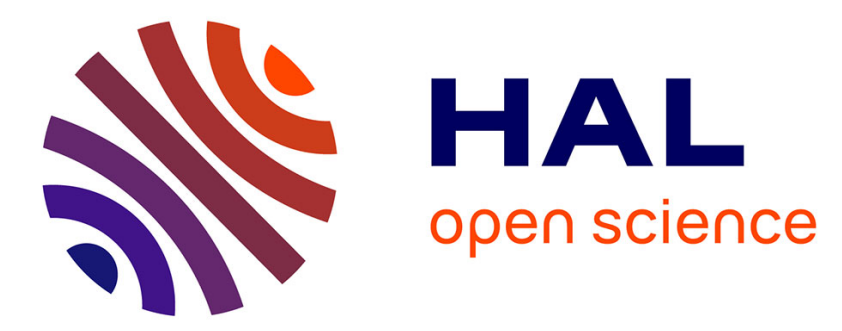

\title{
Saint Venant compatibility equations in curvilinear coordinates
}

\author{
Philippe G. Ciarlet, Cristinel Mardare, Ming Shen
}

\section{To cite this version:}

Philippe G. Ciarlet, Cristinel Mardare, Ming Shen. Saint Venant compatibility equations in curvilinear coordinates. Analysis and Applications, 2007, 5, pp.231-251. hal-00139199

\section{HAL Id: hal-00139199 \\ https://hal.science/hal-00139199}

Submitted on 30 Mar 2007

HAL is a multi-disciplinary open access archive for the deposit and dissemination of scientific research documents, whether they are published or not. The documents may come from teaching and research institutions in France or abroad, or from public or private research centers.
L'archive ouverte pluridisciplinaire HAL, est destinée au dépôt et à la diffusion de documents scientifiques de niveau recherche, publiés ou non, émanant des établissements d'enseignement et de recherche français ou étrangers, des laboratoires publics ou privés. 


\title{
SAINT VENANT EQUATIONS IN CURVILINEAR COORDINATES
}

\author{
PHILIPPE G. CIARLET, CRISTINEL MARDARE AND MING SHEN
}

\begin{abstract}
We establish that the linearized strains in curvilinear coordinates associated with a given displacement field necessarily satisfy "Saint Venant equations in curvilinear coordinates". Furthermore, we show that these equations are also sufficient, in the following sense: If a symmetric matrix field defined over a simplyconnected open set satisfies the Saint Venant equations in curvilinear coordinates, then its coefficients are the linearized strains associated with a displacement field. In addition, our proof provides an explicit algorithm for recovering such a displacement field from its linear strains in curvilinear coordinates. This algorithm may be viewed as the linear counterpart of the reconstruction of an immersion from a given flat Riemannian metric.
\end{abstract}

RÉsumÉ. On établit que le tenseur linéarisé des déformations en coordonnées curvilignes associé à un champ de déplacements donné vérifie nécessairement des "equations de Saint Venant en coordonnées curvilignes". On démontre ensuite que ces équations sont aussi suffisantes, dans le sens suivant: Si un champ de matrices symétriques satisfait les equations de Saint Venant en coordonnées curvilignes dans ouvert simplement connexe, alors il est le tenseur linéarisé des déformations associé à un champ de déplacements. De plus, la preuve fournit un algorithme explicit pour la reconstruction d'un tel champ de déplacements à partir de son tenseur linéarisé des déformations en coordonnées curvilignes. Cet algorithme peut être vu comme une version linéarisée de la reconstruction d'une immersion à partir d'une métrique riemannienne de courbure nulle.

2000 Mathematics Subject Classification. Primary : 49N10. Secondary : 73K15.

Key words and phrases. Differential geometry, Elasticity. 


\section{INTRODUCTION}

All the notations used, but not defined, here are defined in the next sections.

It is well known in differential geometry that a flat Riemannian space can be isometrically immersed, at least locally, in the Euclidean space with the same dimension. For instance, if $\Omega \subset \mathbb{R}^{3}$ is a simply-connected domain endowed with a flat Riemannian metric $\left(g_{i j}\right)$ of class $C^{2}$ in $\Omega$, then there exists an immersion $\Theta: \Omega \rightarrow \mathbb{R}^{3}$ of class $\mathcal{C}^{3}$ in $\Omega$ such that

$$
g_{i j}=\partial_{i} \boldsymbol{\Theta} \cdot \partial_{j} \boldsymbol{\Theta} \text { in } \Omega .
$$

The assumption that the metric $\left(g_{i j}\right)$ is flat means that its Riemannian curvature tensor vanishes in $\Omega$, i.e., that

$$
R_{t k i j}:=g_{t \ell}\left(\partial_{i} \Gamma_{j k}^{\ell}-\partial_{j} \Gamma_{i k}^{\ell}+\Gamma_{j k}^{t} \Gamma_{i t}^{\ell}-\Gamma_{i k}^{t} \Gamma_{j t}^{\ell}\right)=0 \text { in } \Omega,
$$

where

$$
\Gamma_{j k}^{\ell}:=\frac{1}{2} g^{\ell t}\left(\partial_{j} g_{t k}+\partial_{k} g_{t j}-\partial_{t} g_{j k}\right)
$$

denote the Christoffel symbols associated with the metric $\left(g_{i j}\right)$.

The above immersion $\boldsymbol{\Theta}$ is recovered from the metric $\left(g_{i j}\right)$ by first solving the system

$$
\partial_{i} \mathbf{g}_{j}=\Gamma_{i j}^{k} \mathbf{g}_{k} \text { in } \Omega,
$$

with unknowns $\mathbf{g}_{j} \in \mathcal{C}^{2}\left(\Omega ; \mathbb{R}^{3}\right)$, then by solving the system

$$
\partial_{i} \boldsymbol{\Theta}=\mathbf{g}_{i} \text { in } \Omega \text {. }
$$

The mapping $\Theta \in \mathcal{C}^{3}\left(\Omega ; \mathbb{R}^{3}\right)$ found in this fashion is the sought immersion. Note that the first system above has solutions because the Riemannian curvature tensor vanishes in $\Omega$ and that the second system above has solutions because the Christoffel symbols satisfy $\Gamma_{j k}^{\ell}=\Gamma_{k j}^{\ell}$.

Our objective here is to establish an infinitesimal version of this result. More specifically, let there be given a simply-connected domain $\Omega \subset \mathbb{R}^{3}$ and let $\left(e_{i j}\right): \Omega \rightarrow \mathbb{S}^{3}$ be a symmetric matrix field of class $\mathcal{C}^{2}$ in $\Omega$ (this regularity assumption, chosen here for simplicity, will be weakened in the next sections). Then we show that if the matrix field $\left(e_{i j}\right)$ satisfies the "Saint Venant equations in curvilinear coordinates", viz.,

$$
R_{t k i j}^{\operatorname{lin}}:=e_{k i \| j t}+e_{t j \| i k}-e_{k j \| i t}-e_{t i \| j k}=0 \text { in } \Omega,
$$

then there exists a vector field $v: \Omega \rightarrow \mathbb{R}^{3}$ of class $\mathcal{C}^{3}$ such that

$$
e_{i j}=\frac{1}{2}\left(\partial_{i} \boldsymbol{v} \cdot \mathbf{g}_{j}+\mathbf{g}_{i} \cdot \partial_{j} \boldsymbol{v}\right) \text { in } \Omega .
$$

The notation $e_{i j \| k t}$, as well as the notation $e_{i j \| k}$ below, respectively denote the second and the first covariant derivatives of the matrix field $\left(e_{i j}\right)$ (see Section 3 for their explicit expressions). 
The recovery of the vector field $\boldsymbol{v}$ from the matrix field $\left(e_{i j}\right)$ is obtained by first solving the system

$$
a_{i j \| k}=e_{k j \| i}-e_{k i \| j} \text { in } \Omega,
$$

where the unknown is an antisymmetric matrix field $\left(a_{i j}\right) \in \mathcal{C}^{2}\left(\Omega ; \mathbb{A}^{3}\right)$, then by solving the system

$$
\partial_{i} \boldsymbol{v}=\left(e_{i j}+a_{i j}\right) \mathbf{g}^{j} \text { in } \Omega .
$$

The vector field $\boldsymbol{v} \in \mathcal{C}^{3}\left(\Omega ; \mathbb{R}^{3}\right)$ found in this fashion has the desired properties. Note that the first system above has solutions because the Saint Venant equations are satisfied and that the second system above has solutions because the matrix fields $\left(e_{i j}\right)$ and $\left(a_{i j}\right)$ are respectively symmetric and antisymmetric.

This last result may be viewed as an infinitesimal version of the former because the left-hand side of the Saint Venant equations is the linearized part with respect to $\boldsymbol{v}$ of the Riemann curvature tensor associated with the immersion $(\boldsymbol{\Theta}+\boldsymbol{v})$.

The Saint Venant equations derived here in curvilinear coordinates generalize the classical Saint Venant equations in Cartesian coordinates (see, e.g., [2]). In this respect, note that these equations have been likewise extended to "Sant Venant equations on a surface"; see [5].

\section{NotATIONS AND OTHER PRELIMINARIES}

Latin indices and exponents vary in the set $\{1,2,3\}$ and the summation convention with respect to repeated indices and exponents is systematically used in conjunction with this rule.

All spaces, matrices, etc., are real. The Kronecker symbol is denoted $\delta_{i}^{j}$. The symbols $\mathbb{M}^{3}, \mathbb{A}^{3}, \mathbb{S}^{3}$, and $\mathbb{S}_{>}^{3}$ respectively designate the sets of all square matrices of order three, of all antisymmetric matrices of order three, of all symmetric matrices of order three, and of all positive-definite symmetric matrices of order three.

The Euclidean inner product of $\boldsymbol{u}, \boldsymbol{v} \in \mathbb{R}^{3}$ and the Euclidean norm of $\boldsymbol{u} \in \mathbb{R}^{3}$ are denoted by $\boldsymbol{u} \cdot \boldsymbol{v}$ and $|\boldsymbol{u}|$. The notation $\left(t_{i j}\right)$ designates the matrix of $\mathbb{M}^{3}$ with $t_{i j}$ as its elements, the first index $i$ being the row index. The inner-product of two matrices $\left(s_{i j}\right) \in \mathbb{M}^{3}$ and $\left(t_{i j}\right) \in \mathbb{M}^{3}$ is $\sum_{i j} s_{i j} t_{i j}$. The spectral norm of a matrix $\mathbf{A} \in \mathbb{M}^{3}$ is

$$
|\mathbf{A}|:=\sup \left\{|\mathbf{A} \boldsymbol{v}| ; \boldsymbol{v} \in \mathbb{R}^{n},|\boldsymbol{v}| \leq 1\right\} .
$$

The notation $\left.f\right|_{U}$ designates the restriction to a set $U$ of a function $f$, the notation $\boldsymbol{i}_{U}$ designates the identity mapping of the set $U$.

Let $\Omega$ be an open subset of $\mathbb{R}^{3}$. The coordinates of a point $x \in \Omega$ are denoted $x_{i}$. Partial derivative operators of order $m \geq 1$ are denoted

$$
\partial^{\alpha}:=\frac{\partial^{|\alpha|}}{\partial x_{1}^{\alpha_{1}} \partial x_{2}^{\alpha_{2}} \partial x_{3}^{\alpha_{3}}}
$$


where $\alpha=\left(\alpha_{i}\right)$ is a multi-index satisfying $|\alpha|:=\alpha_{1}+\alpha_{2}+\alpha_{3}=m$. Partial derivative operators of the first, second, and third order are also denoted $\partial_{i}:=\partial / \partial x_{i}, \partial_{i j}:=\partial^{2} / \partial x_{i} \partial x_{j}$, and $\partial_{i j k}:=\partial^{3} / \partial x_{i} \partial x_{j} \partial x_{k}$.

The space of all continuous functions from a subset $X \subset \mathbb{R}^{3}$ into a normed space $Y$ is denoted $\mathcal{C}^{0}(X ; Y)$, or simply $\mathcal{C}^{0}(X)$ if $Y=\mathbb{R}$. For any integer $m \geq 1$, the space of all real-valued functions that are $m$ times continuously differentiable in $\Omega$ is denoted $\mathcal{C}^{m}(\Omega)$.

The space $\mathcal{C}^{m}(\bar{\Omega}), m \geq 1$, is defined as that consisting of all vector-valued functions $f \in \mathcal{C}^{1}(\Omega)$ that, together with their partial derivatives of order $\leq m$, possess continuous extentions to the closure $\bar{\Omega}$ of $\Omega$. If $\Omega$ is bounded, then the space $\mathcal{C}^{m}(\bar{\Omega})$ equipped with the norm

$$
\|f\|_{\mathcal{C}^{m}(\bar{\Omega})}:=\max _{|\alpha| \leq m}\left(\sup _{x \in \Omega}\left|\partial^{\alpha} f(x)\right|\right)
$$

is a Banach space. Similar definitions hold for the spaces $\mathcal{C}^{m}\left(\bar{\Omega} ; \mathbb{R}^{3}\right), \mathcal{C}^{m}\left(\bar{\Omega} ; \mathbb{S}^{3}\right)$, etc.

The Lebesgue and Sobolev spaces $L^{p}(\Omega ; Y)$ and $W^{m, p}(\Omega ; Y)$, where $m \geq 1$ is an integer, $p \geq 1$, and $Y$ is one of the spaces $\mathbb{R}, \mathbb{R}^{3}$, or $\mathbb{S}^{3}$, are respectively equipped with the norms

$$
\|f\|_{L^{p}(\Omega ; Y)}:=\left\{\int_{\Omega}|f(x)|^{p} d x\right\}^{1 / p}
$$

and

$$
\|f\|_{W^{m, p}(\Omega ; Y)}:=\left\{\int_{\Omega}\left(|f(x)|^{p}+\sum_{|\alpha| \leq m}\left|\partial^{\alpha} f(x)\right|^{p}\right) d x\right\}^{1 / p} .
$$

We also use the notations $W^{m, p}(\Omega):=W^{m, p}(\Omega ; \mathbb{R})$ and $H^{m}(\Omega ; Y):=W^{m, 2}(\Omega ; Y)$.

The space $W_{\mathrm{loc}}^{m, p}(\Omega ; Y)$ is the space of all mesurable functions such that $f \in W^{m, p}(U ; Y)$ for all bounded open sets $U$ that satisfy $\bar{U} \subset \Omega$.

The space of all indefinitely derivable functions $\varphi: \Omega \rightarrow \mathbb{R}$ with compact support included in $\Omega$ is denoted $\mathcal{D}(\Omega)$ and the space of all distributions over $\Omega$ is denoted $\mathcal{D}^{\prime}(\Omega)$. The closure of $\mathcal{D}(\Omega)$ in $H^{m}(\Omega)$ is denoted $H_{0}^{m}(\Omega)$. Similar definitions hold for the spaces $H_{0}^{m}\left(\Omega ; \mathbb{R}^{3}\right), H_{0}^{m}\left(\Omega ; \mathbb{S}^{3}\right)$, etc. The dual of the space $H_{0}^{m}(\Omega)$ is denoted $H^{-m}(\Omega)$ and the corresponding duality pairing is denoted $\langle\cdot, \cdot\rangle$.

We conclude this section with the following technical result.

Lemma 1. Let $\Omega$ be an open subset of $\mathbb{R}^{3}$.

a) If $f \in \mathcal{C}^{1}(\bar{\Omega})$ and $\chi \in H^{-1}(\Omega)$, then the mapping

$$
\varphi \in H_{0}^{1}(\Omega) \mapsto<\chi, f \varphi>\in \mathbb{R}
$$

belongs to $H^{-1}(\Omega)$ and is denoted $f \chi$.

b) If $f \in \mathcal{C}^{2}(\bar{\Omega})$ and $\chi \in H^{-2}(\Omega)$, then the mapping

$$
\varphi \in H_{0}^{2}(\Omega) \mapsto<\chi, f \varphi>\in \mathbb{R}
$$

belongs to $H^{-2}(\Omega)$ and is denoted $f \chi$. 
Proof. We only need to prove the continuity of the mappings defined in the lemma. If $f \in \mathcal{C}^{1}(\bar{\Omega})$ and $\chi \in H^{-1}(\Omega)$, then there exists a constant $C_{1}$ such that

$$
|<\chi, f \varphi>| \leq\|\chi\|_{H^{-1}(\Omega)}\|f \varphi\|_{H^{1}(\Omega)} \leq C_{1}\|\chi\|_{H^{-1}(\Omega)}\|f\|_{\mathcal{C}^{1}(\bar{\Omega})}\|\varphi\|_{H^{1}(\Omega)}
$$

for all $\varphi \in H_{0}^{1}(\Omega)$. This means that $f \chi \in H^{-1}(\Omega)$.

Likewise, if $f \in \mathcal{C}^{2}(\bar{\Omega})$ and $\chi \in H^{-2}(\Omega)$, then there exists a constant $C_{2}$ such that

$$
|<\chi, f \varphi>| \leq\|\chi\|_{H^{-2}(\Omega)}\|f \varphi\|_{H^{2}(\Omega)} \leq C_{2}\|\chi\|_{H^{-2}(\Omega)}\|f\|_{\mathcal{C}^{2}(\bar{\Omega})}\|\varphi\|_{H^{2}(\Omega)}
$$

for all $\varphi \in H_{0}^{2}(\Omega)$. This means that $f \chi \in H^{-2}(\Omega)$.

Remark. In other words, this lemma asserts that if $f \in \mathcal{C}^{1}(\bar{\Omega})$ and $\chi \in H^{-1}(\Omega)$, then the product $f \chi$ is well defined as an element of $H^{-1}(\Omega)$; and likewise, if $f \in \mathcal{C}^{2}(\bar{\Omega})$ and $\chi \in H^{-2}(\Omega)$, then the product $f \chi$ is well defined as an element of $H^{-2}(\Omega)$.

\section{Curvilinear coordinates and Covariant Derivatives}

A mapping $\boldsymbol{\Theta} \in \mathcal{C}^{1}\left(\bar{\Omega} ; \mathbb{R}^{3}\right)$ is an immersion if the vectors $\partial_{i} \boldsymbol{\Theta}(x)$ are linearly independent at all points $x \in \bar{\Omega}$.

Let $\Omega$ be an open subset of $\mathbb{R}^{3}$ and let there be given an immersion $\Theta \in \mathcal{C}^{3}\left(\bar{\Omega} ; \mathbb{R}^{3}\right)$. Then the invariance of domain theorem shows that the image $\boldsymbol{\Theta}(\Omega)$ is an open set, thus a three-dimensional manifold immersed in $\mathbb{R}^{3}$. For each $x \in \Omega$, the vectors

$$
\mathbf{g}_{i}(x):=\partial_{i} \Theta(x)
$$

form a basis in the tangent space, identified here with $\mathbb{R}^{3}$, to the manifold $\boldsymbol{\Theta}(\Omega)$ at the point $\boldsymbol{\Theta}(x)$. The vector fields $\mathbf{g}^{j}$, defined by

$$
\mathbf{g}_{i}(x) \cdot \mathbf{g}^{j}(x)=\delta_{i}^{j} \text { for all } x \in \Omega,
$$

form the dual basis of the basis formed by the vector fields $\mathbf{g}_{i}$.

The manifold $\boldsymbol{\Theta}(\Omega)$ being naturally endowed with the Euclidean metric inherited from the surrounding space $\mathbb{R}^{3}$, the immersion $\Theta$ induces a Riemannian metric on $\Omega$, defined by its covariant components

$$
g_{i j}(x)=\mathbf{g}_{i}(x) \cdot \mathbf{g}_{j}(x) \text { for all } x \in \Omega .
$$

The contravariant components of this metric are defined by

$$
g^{k \ell}(x)=\mathbf{g}^{k}(x) \cdot \mathbf{g}^{\ell}(x),
$$

or equivalently, by $\left(g^{k \ell}(x)=\left(g_{i j}(x)\right)^{-1}\right.$ for all $x \in \Omega$. In turn, this metric induces the Levi-Civita connection in the manifold $\Omega$, defined by the 
Christoffel symbols

$$
\Gamma_{i j}^{k}:=\frac{1}{2} g^{k \ell}\left(\partial_{i} g_{j \ell}+\partial_{j} g_{i \ell}-\partial_{\ell} g_{i j}\right) \text { in } \Omega .
$$

Note that the regularity assumption on the immersion $\Theta$ implies that $g_{i j}, g^{k \ell} \in$ $\mathcal{C}^{2}(\bar{\Omega})$ and that $\Gamma_{i j}^{k} \in \mathcal{C}^{1}(\bar{\Omega})$. The definition of the Christoffel symbols shows that they satisfy $\Gamma_{i j}^{k}=\Gamma_{j i}^{k}$ and that the derivatives of the vector fields $\mathbf{g}_{i}$ and $\mathbf{g}^{j}$ satisfy

$$
\partial_{k} \mathbf{g}_{i}=\Gamma_{k i}^{\ell} \mathbf{g}_{\ell} \text { and } \partial_{k} \mathbf{g}^{j}=-\Gamma_{k \ell}^{j} \mathbf{g}^{\ell} \text { in } \Omega .
$$

The covariant derivatives of the covariant components $u_{i} \in H^{1}(\Omega)$ of a vector field $u_{i} \mathbf{g}^{i}$ are defined by

$$
u_{j \| i}:=\partial_{i} u_{j}-\Gamma_{i j}^{k} u_{k},
$$

or, equivalently, by

$$
u_{j \| i} \mathbf{g}^{j}=\partial_{i}\left(u_{j} \mathbf{g}^{j}\right) .
$$

The covariant derivatives of a second-order tensor field with covariant components $T_{i j} \in L^{2}(\Omega)$ are defined by

$$
T_{i j \| k}:=\partial_{k} T_{i j}-\Gamma_{k i}^{\ell} T_{\ell j}-\Gamma_{k j}^{\ell} T_{i \ell}
$$

and they belong to the space $H^{-1}(\Omega)$ (cf. Lemma 1 ). Since the matrix fields

$$
\mathbf{g}^{i} \otimes \mathbf{g}^{j}:=\mathbf{g}^{i}\left(\mathbf{g}^{j}\right)^{T}
$$

form a basis in the space $\mathcal{C}^{2}\left(\bar{\Omega} ; \mathbb{M}^{3}\right)$ and since

$$
\partial_{k}\left(\mathbf{g}^{i} \otimes \mathbf{g}^{j}\right)=-\Gamma_{k \ell}^{i} \mathbf{g}^{\ell} \otimes \mathbf{g}^{j}-\Gamma_{k \ell}^{j} \mathbf{g}^{i} \otimes \mathbf{g}^{\ell},
$$

the above definition of the covariant derivatives $T_{i j \| k}$ shows that

$$
T_{i j \| k} \mathbf{g}^{i} \otimes \mathbf{g}^{j}=\partial_{k}\left(T_{i j} \mathbf{g}^{i} \otimes \mathbf{g}^{j}\right) .
$$

Note that such equations are to be understood in the distributional sense, the functions $T_{i j}$ being only in $L^{2}(\Omega)$.

Finally, for all third-order tensor field with covariant components $T_{i j k} \in$ $H^{-1}(\Omega)$, we define the covariant derivatives

$$
T_{i j k \| \ell}:=\partial_{\ell} T_{i j k}-\Gamma_{\ell i}^{t} T_{t j k}-\Gamma_{\ell j}^{t} T_{i t k}-\Gamma_{\ell k}^{t} T_{i j t},
$$

which, in view of Lemma 1 , are well defined as distributions in $H^{-2}(\Omega)$.

If $T_{i j} \in L^{2}(\Omega)$, the second-order covariant derivatives $T_{i j \| k \ell}$ are defined by the relations

$$
T_{i j \| k \ell}:=\partial_{\ell} T_{i j \| k}-\Gamma_{\ell i}^{t} T_{t j \| k}-\Gamma_{\ell j}^{t} T_{i t \| k}-\Gamma_{\ell k}^{t} T_{i j \| t} .
$$

It is then easily seen, in view of relation (1), that these second-order covariant derivatives satisfy

$$
T_{i j \| k \ell} \mathbf{g}^{i} \otimes \mathbf{g}^{j}=\left(\partial_{\ell k}-\Gamma_{\ell k}^{t} \partial_{t}\right)\left(T_{i j} \mathbf{g}^{i} \otimes \mathbf{g}^{j}\right) .
$$


Indeed, relation (1) implies that

$$
\begin{aligned}
\partial_{\ell k}\left(T_{i j} \mathbf{g}^{i} \otimes \mathbf{g}^{j}\right) & =\partial_{\ell}\left(T_{i j \| k} \mathbf{g}^{i} \otimes \mathbf{g}^{j}\right) \\
& =\left(\partial_{\ell} T_{i j \| k}\right) \mathbf{g}^{i} \otimes \mathbf{g}^{j}-\left(\Gamma_{\ell t}^{i} T_{i j \| k}\right) \mathbf{g}^{t} \otimes \mathbf{g}^{j}-\left(\Gamma_{\ell t}^{j} T_{i j \| k}\right) \mathbf{g}^{i} \otimes \mathbf{g}^{t} \\
& =\left(\partial_{\ell} T_{i j \| k}-\Gamma_{\ell i}^{t} T_{t j \| k}-\Gamma_{\ell j}^{t} T_{i t \| k}\right) \mathbf{g}^{i} \otimes \mathbf{g}^{j} \\
& =\left(T_{i j \| k \ell}+\Gamma_{\ell k}^{t} T_{i j \| t}\right) \mathbf{g}^{i} \otimes \mathbf{g}^{j} \\
& =\left(T_{i j \| k \ell}\right) \mathbf{g}^{i} \otimes \mathbf{g}^{j}+\Gamma_{\ell k}^{t} \partial_{t}\left(T_{i j} \mathbf{g}^{i} \otimes \mathbf{g}^{j}\right) .
\end{aligned}
$$

Note that relation (2) implies in particular that the second-order covariant derivatives satisfy:

$$
T_{i j \| k \ell}=T_{i j \| \ell k}
$$

\section{Poincaré Theorem in Curvilinear coordinates}

In what follows, a domain in $\mathbb{R}^{3}$ is a bounded and connected open set with a Lipschitz-continuous boundary, the set $\omega$ being locally on the same side of its boundary. The definition of such a boundary is the usual one, as found for instance in Adams [1], Grisvard [6], or Nečas [7].

Poincaré Theorem, which is classically proved only for continuously differentiable functions, was generalized by Ciarlet \& Ciarlet, Jr. [2] into the following.

Theorem 1. Let $\Omega$ be a simply connected domain of $\mathbb{R}^{3}$. Let $h_{k} \in H^{-1}(\Omega)$ be distributions that satisfy

$$
\partial_{\ell} h_{k}=\partial_{k} h_{\ell} \text { in } H^{-2}(\Omega) .
$$

Then there exists a function $p \in L^{2}(\Omega)$, unique up to an additive constant, such that

$$
h_{k}=\partial_{k} p \text { in } H^{-1}(\Omega) .
$$

Clearly, this theorem remains valid if the functions $h_{k}$ are replaced with matrix fields with components in $H^{-1}(\Omega)$, the function $p$ being then replaced by a matrix field with components in $L^{2}(\Omega)$.

We now show that a similar result holds in curvilinear coordinates.

Theorem 2. Let $\Omega$ be a simply connected domain of $\mathbb{R}^{3}$ and let $\boldsymbol{\Theta} \in$ $\mathcal{C}^{3}\left(\bar{\Omega} ; \mathbb{R}^{3}\right)$ be an immersion. Let $h_{i j k} \in H^{-1}(\Omega)$ be distributions that satisfy

$$
h_{i j k \| \ell}=h_{i j \ell \| k} \text { in } H^{-2}(\Omega) .
$$

Then there exists functions $p_{i j} \in L^{2}(\Omega)$, unique up to additive constants, such that

$$
h_{i j k}=p_{i j \| k} \text { in } H^{-1}(\Omega) .
$$

Proof. Define the matrix fields

$$
\mathbf{g}^{i} \otimes \mathbf{g}^{j}:=\mathbf{g}^{i}\left(\mathbf{g}^{j}\right)^{T} \in \mathcal{C}^{1}\left(\bar{\Omega} ; \mathbb{M}^{3}\right),
$$


and note that $\left\{\mathbf{g}^{i}(x) \otimes \mathbf{g}^{j}(x)\right\}$ form a basis in $\mathbb{M}^{3}$ for all $x \in \bar{\Omega}$. Its dual basis with respect to the usual inner product of matrices is $\left\{\mathbf{g}_{i}(x) \otimes \mathbf{g}_{j}(x)\right\}$, where

$$
\mathbf{g}_{i} \otimes \mathbf{g}_{j}:=\mathbf{g}_{i}\left(\mathbf{g}_{j}\right)^{T} \text { in } \Omega .
$$

Let the matrix fields $\mathbf{H}_{k}$ be defined by

$$
\mathbf{H}_{k}:=h_{i j k} \mathbf{g}^{i} \otimes \mathbf{g}^{j} .
$$

The derivatives in the distributional sense of the fields $\mathbf{H}_{k}$, which belong to $H^{-1}\left(\Omega ; \mathbb{M}^{3}\right)$ by Lemma 1 , are given by

$$
\begin{aligned}
\partial_{\ell} \mathbf{H}_{k} & =\left(\partial_{\ell} h_{i j k}\right) \mathbf{g}^{i} \otimes \mathbf{g}^{j}+h_{i j k} \partial_{\ell}\left(\mathbf{g}^{i} \otimes \mathbf{g}^{j}\right) \\
& =\left(\partial_{\ell} h_{i j k}\right) \mathbf{g}^{i} \otimes \mathbf{g}^{j}-h_{i j k}\left(\Gamma_{\ell s}^{i} \mathbf{g}^{s} \otimes \mathbf{g}^{j}+\Gamma_{\ell s}^{j} \mathbf{g}^{i} \otimes \mathbf{g}^{s}\right) \\
& =\left(\partial_{\ell} h_{i j k}-\Gamma_{\ell i}^{s} h_{s j k}-\Gamma_{\ell j}^{s} h_{i s k}\right) \mathbf{g}^{i} \otimes \mathbf{g}^{j} \operatorname{in} H^{-2}(\Omega) .
\end{aligned}
$$

Using the definition of covariant derivatives, the above expressions read

$$
\partial_{\ell} \mathbf{H}_{k}=\left(h_{i j k \| \ell}+\Gamma_{\ell k}^{s} h_{i j s}\right) \mathbf{g}^{i} \otimes \mathbf{g}^{j} \text { in } H^{-2}(\Omega) .
$$

Hence assumption (3) shows that

$$
\partial_{\ell} \mathbf{H}_{k}=\partial_{k} \mathbf{H}_{\ell} \text { in } H^{-2}\left(\Omega ; \mathbb{M}^{3}\right) .
$$

Then Theorem 1 shows that there exists a matrix field $\mathbf{P} \in L^{2}\left(\Omega ; \mathbb{M}^{3}\right)$, unique up to additive constant matrix field, such that

$$
\mathbf{H}_{k}=\partial_{k} \mathbf{P} \text { in } H^{-1}\left(\Omega ; \mathbb{M}^{3}\right) .
$$

Since $\left\{\mathbf{g}^{i}(x) \otimes \mathbf{g}^{j}(x)\right\}$ form a basis in $\mathbb{M}^{3}$ for all $x \in \Omega$, the matrix field $\mathbf{P}$ can be expanded over this basis as

$$
\mathbf{P}=p_{i j} \mathbf{g}^{i} \otimes \mathbf{g}^{j} \text { in } \Omega,
$$

and since $p_{i j}=\operatorname{tr}\left(\mathbf{P}^{T}\left(\mathbf{g}_{i} \otimes \mathbf{g}_{j}\right)\right)$, we clearly have $p_{i j} \in L^{2}(\Omega)$.

Hence the matrix fields $\mathbf{H}_{k}$ can be re-written as

$$
\mathbf{H}_{k}=h_{i j k} \mathbf{g}^{i} \otimes \mathbf{g}^{j}=\partial_{k}\left(p_{i j} \mathbf{g}^{i} \otimes \mathbf{g}^{j}\right) .
$$

But the definition of the covariant derivative shows that

$$
\partial_{k}\left(p_{i j} \mathbf{g}^{i} \otimes \mathbf{g}^{j}\right)=p_{i j \| k} \mathbf{g}^{i} \otimes \mathbf{g}^{j} .
$$

Combining now the last two equations, we finally find that

$$
h_{i j k}=p_{i j \| k} \text {. }
$$

That the solution $p_{i j}$ is unique up to additive constants is clear.

Remark. Theorem 2 can also be established as a consequence of Theorem A.4 of [10] establishing the existence of solutions to Pfaff systems, of which the equations $p_{i j \|_{k}}=h_{i j k}$ constitutes a special case. 


\section{SAint Venant EQUATIONS in CURVILINEAR COORDinateS}

Let $\Omega$ be a bounded open subset of $\mathbb{R}^{3}$ and let $\Theta \in \mathcal{C}^{3}\left(\bar{\Omega} ; \mathbb{R}^{3}\right)$ be an immersion. As in Section 3, let the vector fields $\mathbf{g}_{i} \in \mathcal{C}^{2}\left(\bar{\Omega} ; \mathbb{R}^{3}\right)$ and $\mathbf{g}^{i} \in$ $\mathcal{C}^{2}\left(\bar{\Omega} ; \mathbb{R}^{3}\right)$ be defined by

$$
\mathbf{g}_{i}:=\partial_{i} \boldsymbol{\Theta} \text { and } \mathbf{g}_{i} \cdot \mathbf{g}^{j}=\delta_{i}^{j} .
$$

With every vector field $\boldsymbol{u} \in H^{1}\left(\Omega ; \mathbb{R}^{3}\right)$, we associate the linearized strains in curvilinear coordinates, also known as the covariant components of the linearized change of metric tensor, defined by

$$
\varepsilon_{i j}(\boldsymbol{u}):=\frac{1}{2}\left(\partial_{i} \boldsymbol{u} \cdot \mathbf{g}_{j}+\mathbf{g}_{i} \cdot \partial_{j} \boldsymbol{u}\right) .
$$

Note that $\varepsilon_{i j}(\boldsymbol{u}) \in L^{2}(\Omega)$ for all $i, j$ and that $\varepsilon_{i j}(\boldsymbol{u})=\varepsilon_{j i}(\boldsymbol{u})$.

The next theorem shows that the functions $\varepsilon_{i j}(\boldsymbol{u})$ satisfy crucial compatibility relations, which we will call the Saint Venant equations in curvilinear coordinates since they generalize the well-known saint Venant equations in Cartesian coordinates.

Theorem 3. The linearized strains in curvilinear coordinates $\varepsilon_{i j}(\boldsymbol{u}) \in$ $L^{2}(\Omega)$ associated with a vector field $\boldsymbol{u} \in H^{1}\left(\Omega ; \mathbb{R}^{3}\right)$ satisfy the relations

$$
\varepsilon_{k i \| j \ell}(\boldsymbol{u})+\varepsilon_{\ell j \| i k}(\boldsymbol{u})-\varepsilon_{k j \| i \ell}(\boldsymbol{u})-\varepsilon_{\ell i \| j k}(\boldsymbol{u})=0 \text { in } H^{-2}(\Omega) .
$$

Proof. Given a vector field $\boldsymbol{u} \in H^{1}\left(\Omega ; \mathbb{R}^{3}\right)$, let

$$
e_{i j}:=\varepsilon_{i j}(\boldsymbol{u}) \in L^{2}(\Omega)
$$

and

$$
a_{i j}:=\frac{1}{2}\left(\partial_{i} \boldsymbol{u} \cdot \mathbf{g}_{j}-\mathbf{g}_{i} \cdot \partial_{j} \boldsymbol{u}\right) \in L^{2}(\Omega),
$$

i.e., $e_{i j}$ and $a_{i j}$ are respectively the symmetric and the antisymmetric parts of the tensor $u_{i \| j}$. The derivatives in the distributional sense of the vector field $\boldsymbol{u}$ are then given by

$$
\partial_{i} \boldsymbol{u}=\left(\partial_{i} \boldsymbol{u} \cdot \mathbf{g}_{j}\right) \mathbf{g}^{j}=\left(e_{i j}+a_{i j}\right) \mathbf{g}^{j}
$$

in $L^{2}\left(\Omega ; \mathbb{R}^{3}\right)$, which shows that these derivatives are completely determined by the symmetric tensor $e_{i j}$ and the antisymmetric tensor $a_{i j}$. In fact, they are determined only by the tensor $e_{i j}$, as we now show. Since $\partial_{i} \mathbf{g}_{j}=\partial_{j} \mathbf{g}_{i}$, we first have

$$
\begin{aligned}
2 \partial_{k} a_{i j} & =\partial_{k i} \boldsymbol{u} \cdot \mathbf{g}_{j}+\partial_{i} \boldsymbol{u} \cdot \partial_{k} \mathbf{g}_{j}-\partial_{k j} \boldsymbol{u} \cdot \mathbf{g}_{i}-\partial_{j} \boldsymbol{u} \cdot \partial_{k} \mathbf{g}_{i} \\
& =\partial_{i}\left(2 e_{k j}-\partial_{j} \boldsymbol{u} \cdot \mathbf{g}_{k}\right)-\partial_{j}\left(2 e_{k i}-\partial_{i} \boldsymbol{u} \cdot \mathbf{g}_{k}\right)+\partial_{i} \boldsymbol{u} \cdot \partial_{k} \mathbf{g}_{j}-\partial_{j} \boldsymbol{u} \cdot \partial_{k} \mathbf{g}_{i} \\
& =2\left(\partial_{i} e_{k j}-\partial_{j} e_{k i}+\partial_{i} \boldsymbol{u} \cdot \partial_{j} \mathbf{g}_{k}-\partial_{j} \boldsymbol{u} \cdot \partial_{i} \mathbf{g}_{k}\right),
\end{aligned}
$$

all equalities being valid in the distributional sense. Combining this last equality with the relations

$$
\begin{aligned}
& \partial_{i} \boldsymbol{u} \cdot \partial_{j} \mathbf{g}_{k}=\Gamma_{j k}^{\ell}\left(\partial_{i} \boldsymbol{u} \cdot \mathbf{g}_{\ell}\right)=\Gamma_{j k}^{\ell}\left(e_{i \ell}+a_{i \ell}\right), \\
& \partial_{j} \boldsymbol{u} \cdot \partial_{i} \mathbf{g}_{k}=\Gamma_{i k}^{\ell}\left(\partial_{j} \boldsymbol{u} \cdot \mathbf{g}_{\ell}\right)=\Gamma_{i k}^{\ell}\left(e_{j \ell}+a_{j \ell}\right),
\end{aligned}
$$


we next deduce that

$$
\begin{aligned}
\partial_{k} a_{i j}-\Gamma_{j k}^{\ell} a_{i \ell}+\Gamma_{i k}^{\ell} a_{j \ell} & =\left(\partial_{i} e_{k j}-\Gamma_{i k}^{\ell} e_{j \ell}\right)-\left(\partial_{j} e_{k i}-\Gamma_{j k}^{\ell} e_{i \ell}\right) \\
& =e_{k j \| i}-e_{k i \| j}
\end{aligned}
$$

But the first term is equal to the covariant derivative $a_{i j \| k}$, since $a_{j \ell}=-a_{\ell j}$. Hence the previous equality becomes

$$
a_{i j \| k}=e_{k j \| i}-e_{k i \| j} .
$$

Then the relations (see the Section 3)

$$
a_{i j \| k \ell}=a_{i j \| \ell k} \text { in } H^{-2}(\Omega),
$$

imply that

$$
e_{k j \| i \ell}-e_{k i \| j \ell}=e_{\ell j \| i k}-e_{\ell i \| j k} \text { in } H^{-2}(\Omega) .
$$

This means that the Saint Venant equations (4) are satisfied in the distributional sense.

Remarks. (1) Equation (5) shows that the antisymmetric matrix field $\left(a_{i j}\right)$ is uniquely determined by the linear strains $\varepsilon_{i j}(\boldsymbol{u})$ up to an antisymmetric matrix field that is constant in each connected component of $\Omega$.

(2) Equation (6) shows that the Saint Venant equations in curvilinear coordinates simply express that $u_{i \| j k \ell}=u_{i \| j \ell k}$. To see this, we note that

$$
u_{i \| j}=e_{i j}+a_{i j},
$$

relations which combined with relations (5) show that

$$
u_{i \| j k \ell}=e_{i j \| k \ell}+e_{k j \| i \ell}-e_{k i \| j \ell} .
$$

Hence the Saint Venant equations hold true if and only if $u_{i \| j k \ell}=u_{i \| j \ell k}$. These relations are also equivalent with the relations

$$
\partial_{i} \boldsymbol{u}\left\|_{k \ell}=\partial_{i} \boldsymbol{u}\right\|_{\ell k},
$$

where the second-order covariant derivatives of the vector fields $\partial_{i} \boldsymbol{u} \in$ $L^{2}\left(\Omega ; \mathbb{R}^{3}\right)$ are defined by replacing $T_{i}$ with $\partial_{i} \boldsymbol{u}$ in the definition of the secondorder covariant derivatives of first-order tensor field with covariant components $T_{i}$. More specifically, if $\boldsymbol{v}_{i}:=\partial_{i} \boldsymbol{u} \in L^{2}\left(\Omega ; \mathbb{R}^{3}\right)$, then

$$
\begin{aligned}
\boldsymbol{v}_{i} \|_{k} & :=\partial_{k} \boldsymbol{v}_{i}-\Gamma_{k i}^{r} \boldsymbol{v}_{r} \text { in } H^{-1}\left(\Omega ; \mathbb{R}^{3}\right), \\
\boldsymbol{v}_{i} \|_{k \ell} & :=\left(\boldsymbol{v}_{i} \|_{k}\right)\left\|_{\ell}=\partial_{\ell} \boldsymbol{v}_{i}\right\|_{k}-\Gamma_{\ell i}^{r} \boldsymbol{v}_{r}\left\|_{k}-\Gamma_{\ell k}^{r} \boldsymbol{v}_{i}\right\|_{r} \text { in } H^{-2}\left(\Omega ; \mathbb{R}^{3}\right) .
\end{aligned}
$$




\section{RECOVERY OF A VECTOR FIELD FROM THE ASSOCIATED LINEARIZED CHANGE OF METRIC TENSOR}

Let $\Omega$ be a bounded and open subset of $\mathbb{R}^{3}$ and let $\Theta \in \mathcal{C}^{3}\left(\bar{\Omega} ; \mathbb{R}^{3}\right)$ be an immersion. As in Section 3, the vectors fields $\mathbf{g}_{i}$ and $\mathbf{g}^{j}$ are defined by

$$
\mathbf{g}_{i}(x):=\partial_{i} \boldsymbol{\Theta}(x) \text { and } \mathbf{g}_{i}(x) \cdot \mathbf{g}^{j}(x)=\delta_{i}^{j} \text { for all } x \in \bar{\Omega},
$$

the covariant components of the Riemannian metric induced in $\Omega$ by the immersion $\Theta$ are defined by

$$
g_{i j}(x)=\mathbf{g}_{i}(x) \cdot \mathbf{g}_{j}(x),
$$

the contravariant components of the same metric are defined by

$$
g^{i j}(x)=\mathbf{g}^{i}(x) \cdot \mathbf{g}^{j}(x),
$$

and the Christoffel symbols associated with the metric $\left(g_{i j}\right)$ are defined by

$$
\Gamma_{i j}^{k}:=\frac{1}{2} g^{k \ell}\left(\partial_{i} g_{j \ell}+\partial_{j} g_{i \ell}-\partial_{\ell} g_{i j}\right) .
$$

Finally, the second-order covariant derivatives of a matrix field $e_{i j} \mathbf{g}^{i} \otimes \mathbf{g}^{j}$ with $e_{i j} \in L^{2}(\Omega)$ are defined by

$$
e_{i j \| k \ell}:=\partial_{\ell} e_{i j \| k}-\Gamma_{\ell i}^{t} e_{t j \| k}-\Gamma_{\ell j}^{t} e_{i t \| k}-\Gamma_{\ell k}^{t} e_{i j \| t},
$$

where

$$
e_{i j \| k}:=\partial_{k} e_{i j}-\Gamma_{k i}^{t} e_{t j}-\Gamma_{k j}^{t} e_{i t}
$$

are the (first-order) covariant derivatives of the same matrix field.

We are now in a position to characterize the space of all symmetric matrix fields that satisfy the Saint Venant equations in curvilinear coordinates:

Theorem 4. Let $\Omega$ be a simply-connected domain in $\mathbb{R}^{3}$ and let $\boldsymbol{\Theta} \in$ $\mathcal{C}^{3}\left(\bar{\Omega} ; \mathbb{R}^{3}\right)$ be an immersion. Let there be given a symmetric matrix field $\left(e_{i j}\right) \in L^{2}\left(\Omega ; \mathbb{S}^{3}\right)$ that satisfies the Saint Venant equations in curvilinear coordinates

$$
e_{k i \| j \ell}+e_{\ell j \| i k}-e_{k j \| i \ell}-e_{\ell i \| j k}=0 \text { in } H^{-2}(\Omega) .
$$

Then there exists a vector field $\boldsymbol{v} \in H^{1}\left(\Omega ; \mathbb{R}^{3}\right)$ such that

$$
e_{i j}=\frac{1}{2}\left(\partial_{i} \boldsymbol{v} \cdot \mathbf{g}_{j}+\mathbf{g}_{i} \cdot \partial_{j} \boldsymbol{v}\right) \text { in } L^{2}\left(\Omega ; \mathbb{S}^{3}\right) .
$$

Proof. The proof consists in first finding an antisymmetric matrix field $\left(a_{i j}\right) \in L^{2}\left(\Omega ; \mathbb{A}^{3}\right)$ that satisfies the equations

$$
a_{i j \| k}=e_{k j \| i}-e_{k i \| j} \text { in } H^{-1}(\Omega),
$$

then in solving in $H^{1}\left(\Omega ; \mathbb{R}^{3}\right)$ the system

$$
\partial_{i} \boldsymbol{v}=\left(e_{i j}+a_{i j}\right) \mathbf{g}^{j} \text { in } L^{2}\left(\Omega ; \mathbb{R}^{3}\right),
$$

the field $\boldsymbol{v}$ being that announced in the statement of the theorem. 
We now begin the detailed proof. Since the equations (7) are satisfied, Theorem 2 shows that there exist functions $\tilde{a}_{i j} \in L^{2}(\Omega)$, unique up to additive constants, such that

$$
\tilde{a}_{i j \| k}=e_{k j \| i}-e_{k i \| j} \text { in } H^{-1}(\Omega) .
$$

Since the right-hand side of this equation is antisymmetric in $(i, j)$, we deduce that

$$
\tilde{a}_{i j \| k}+\tilde{a}_{j i \| k}=0 \text { in } H^{-1}(\Omega) .
$$

Therefore, again by Theorem 2 , there exist constants $c_{i j}$ such that

$$
\tilde{a}_{i j}(x)+\tilde{a}_{j i}(x)=c_{i j} \text { for almost all } x \in \Omega .
$$

Noting that the constants $c_{i j}$ must be symmetric in $(i, j)$, we deduce that the functions

$$
a_{i j}:=\tilde{a}_{i j}+\frac{1}{2} c_{i j}
$$

are antisymmetric in $(i, j)$, belong to the space $L^{2}(\Omega)$, and satisfy the equations

$$
a_{i j \| k}=e_{k j \| i}-e_{k i \| j} .
$$

Let us now prove that there exists a solution $\boldsymbol{v} \in H^{1}\left(\Omega ; \mathbb{R}^{3}\right)$ to the system

$$
\partial_{i} \boldsymbol{v}=\left(e_{i j}+a_{i j}\right) \mathbf{g}^{j} .
$$

To this end, we need to prove that

$$
\partial_{k}\left(\left(e_{i j}+a_{i j}\right) \mathbf{g}^{j}\right)=\partial_{i}\left(\left(e_{k j}+a_{k j}\right) \mathbf{g}^{j}\right) .
$$

Since

$$
\begin{aligned}
\partial_{k}\left(\left(e_{i j}+a_{i j}\right) \mathbf{g}^{j}\right) & =\partial_{k}\left(e_{i j}+a_{i j}\right) \mathbf{g}^{j}-\left(e_{i j}+a_{i j}\right) \Gamma_{k \ell}^{j} \mathbf{g}^{\ell} \\
& =\left(\partial_{k} e_{i \ell}-\Gamma_{k \ell}^{j} e_{i j}\right) \mathbf{g}^{\ell}+\left(\partial_{k} a_{i \ell}-\Gamma_{k \ell}^{j} a_{i j}\right) \mathbf{g}^{\ell} \\
& =\left(e_{i \ell \| k}+\Gamma_{k i}^{j} e_{j \ell}\right) \mathbf{g}^{\ell}+\left(a_{i \ell \| k}+\Gamma_{k i}^{j} a_{j \ell}\right) \mathbf{g}^{\ell},
\end{aligned}
$$

it suffices to prove the equality

$$
\left(e_{i \ell \| k}+\Gamma_{k i}^{j} e_{j \ell}\right)+\left(a_{i \ell \| k}+\Gamma_{k i}^{j} a_{j \ell}\right)=\left(e_{k \ell \| i}+\Gamma_{i k}^{j} e_{j \ell}\right)+\left(a_{k \ell \| i}+\Gamma_{i k}^{j} a_{j \ell}\right) .
$$

Since $\Gamma_{k i}^{j}=\Gamma_{i k}^{j}$, this equality becomes

$$
e_{i \ell \| k}+a_{i \ell \| k}=e_{k \ell \| i}+a_{k \ell \| i}
$$

which, in view of (8), is equivalent with

$$
e_{i \ell \| k}+e_{k \ell \| i}-e_{k i \| \ell}=e_{k \ell \| i}+e_{i \ell \| k}-e_{i k \| \ell} .
$$

But this last equation is clearly satisfied, since the matrix field $\left(e_{i j}\right)$ is symmetric. Hence Theorem 1 shows that there exists a field $\boldsymbol{v} \in L^{2}\left(\Omega ; \mathbb{R}^{3}\right)$, unique up to an additive constant vector field, that satisfies the system

$$
\partial_{i} \boldsymbol{v}=\left(e_{i j}+a_{i j}\right) \mathbf{g}^{j} .
$$

Since the right-hand side of this system belongs in fact to $L^{2}\left(\Omega ; \mathbb{R}^{3}\right)$, the field $\boldsymbol{v}$ belongs to the space $H^{1}\left(\Omega ; \mathbb{R}^{3}\right)$. 
That the vector field $\boldsymbol{v}$ does indeed satisfy the required equations is a consequence of the symmetry of the matrix field $\left(e_{i j}\right)$ and of the anti-symmetry of the matrix field $\left(a_{i j}\right)$, which together show that

$$
\frac{1}{2}\left(\partial_{i} \boldsymbol{v} \cdot \mathbf{g}_{j}+\mathbf{g}_{i} \cdot \partial_{j} \boldsymbol{v}\right)=\frac{1}{2}\left(e_{i j}+a_{i j}+e_{j i}+a_{j i}\right)=e_{i j} .
$$

Remark. Theorem 3 of Ciarlet \& C. Mardare [3] shows that, if the open set $\Omega$ is connected, any other vector field $\tilde{\boldsymbol{v}} \in H^{1}\left(\Omega ; \mathbb{R}^{3}\right)$ that satisfies

$$
e_{i j}=\frac{1}{2}\left(\partial_{i} \tilde{\boldsymbol{v}} \cdot \mathbf{g}_{j}+\mathbf{g}_{i} \cdot \partial_{j} \tilde{\boldsymbol{v}}\right) \text { in } L^{2}\left(\Omega ; \mathbb{S}^{3}\right)
$$

is necessarily of the form

$$
\tilde{\boldsymbol{v}}(x)=\boldsymbol{v}(x)+(\mathbf{a}+\mathbf{b} \wedge \boldsymbol{\Theta}(x)) \text { for almost all } x \in \Omega,
$$

where $\mathbf{a}$ and $\mathbf{b}$ are vectors in $\mathbb{R}^{3}$.

\section{The Riemann curvature tensor and the Saint Venant EQUATIONS}

The objective of this Section is to show that the Saint Venant equations in curvilinear coordinates are nothing but an infinitesimal version of the compatibility conditions that a three-dimensional Riemannian space must satisfy in order to be isometrically immersed in the three-dimensional Euclidean space. These compatibility conditions are recalled in the next theorem, which is a straighforward extension of a well-known result in differential geometry, classicaly established only for smooth immersions $\Theta \in \mathcal{C}^{3}\left(\bar{\Omega} ; \mathbb{R}^{3}\right)$.

Theorem 5. Let $\Omega$ be an open subset of $\mathbb{R}^{3}$ and let $p>3$. Given any immersion $\boldsymbol{\Theta} \in W_{\mathrm{loc}}^{2, p}\left(\Omega ; \mathbb{R}^{3}\right)$, let the functions $g_{i j} \in W_{\mathrm{loc}}^{1, p}\left(\Omega ; \mathbb{S}_{>}^{3}\right)$ be defined by

$$
g_{i j}=\partial_{i} \Theta \cdot \partial_{j} \Theta \text { in } \Omega .
$$

Then the Riemann curvature tensor associated with the matrix field $\left(g_{i j}\right)$ vanishes in the distributional sense, i.e.,

$$
R_{s k i j}:=g_{s \ell}\left(\partial_{i} \Gamma_{j k}^{\ell}-\partial_{j} \Gamma_{i k}^{\ell}+\Gamma_{j k}^{r} \Gamma_{i r}^{\ell}-\Gamma_{i k}^{r} \Gamma_{j r}^{\ell}\right)=0 \text { in } \mathcal{D}^{\prime}(\Omega) .
$$

Proof. Since $W_{\text {loc }}^{1, p}(\Omega) \subset \mathcal{C}^{0}(\Omega)$ by the Sobolev embedding theorem and since $\operatorname{det}\left(g_{i j}\right)>0$ in $\Omega$ (the matrix $\left(g_{i j}(x)\right)$ being positive definite for all $x \in \Omega$ by assumption), the definition of the inverse of a matrix shows that $\left(g^{k \ell}\right)=\left(g_{i j}\right)^{-1} \in W_{\text {loc }}^{1, p}\left(\Omega ; \mathbb{S}_{>}^{3}\right)$. Hence the Christoffel symbols

$$
\Gamma_{j k}^{\ell}:=\frac{1}{2} g^{\ell r}\left(\partial_{j} g_{r k}+\partial_{k} g_{r j}-\partial_{r} g_{j k}\right)
$$

belong to the space $L_{\text {loc }}^{p}(\Omega)$. 
Let the vectors fields $\mathbf{g}_{i}$ and $\mathbf{g}^{j}$ by defined by

$$
\mathbf{g}_{i}:=\partial_{i} \boldsymbol{\Theta} \text { and } \mathbf{g}_{i} \cdot \mathbf{g}^{j}=\delta_{i}^{j} \text { in } \Omega .
$$

Then we deduce from the relations (9) that

$$
\partial_{j} g_{r k}=\partial_{j} \mathbf{g}_{r} \cdot \mathbf{g}_{k}+\mathbf{g}_{r} \cdot \partial_{j} \mathbf{g}_{k}
$$

relation which combined with the above definition of the Christoffel symbols implies that

$$
\Gamma_{j k}^{\ell}=g^{\ell r}\left(\mathbf{g}_{r} \cdot \partial_{j} \mathbf{g}_{k}\right) .
$$

Since $\mathbf{g}^{\ell}=g^{\ell k} \mathbf{g}_{k}$ and since the vectors $\left\{\mathbf{g}_{i}(x)\right\}$ form a basis in $\mathbb{R}^{3}$ for all $x \in \Omega$, we next deduce that

$$
\partial_{j} \mathbf{g}_{k}=\Gamma_{j k}^{\ell} \mathbf{g}_{\ell} \text { in } L_{\mathrm{loc}}^{p}\left(\Omega ; \mathbb{R}^{3}\right) .
$$

Using now Schwarz lemma applied to the second derivatives of $\mathbf{g}_{k}$ in the space of distributions, we next infer from the above relation that

$$
\partial_{i}\left(\Gamma_{j k}^{\ell} \mathbf{g}_{\ell}\right)=\partial_{j}\left(\Gamma_{i k}^{\ell} \mathbf{g}_{\ell}\right)
$$

for all $i, j, k, \ell$ in the distributional sense. Combining the last two relations then shows that

$$
\partial_{i} \Gamma_{j k}^{\ell}-\partial_{j} \Gamma_{i k}^{\ell}+\Gamma_{j k}^{r} \Gamma_{i r}^{\ell}-\Gamma_{i k}^{r} \Gamma_{j r}^{\ell}=0
$$

in the distributional sense, which means that the Riemann curvature tensor of the metric $g_{i j}$ vanishes in $\Omega$.

Remarkably, the converse of Theorem 5 is also true, but inevitable, under slightly more restrictive assumptions:

Theorem 6. Let $\Omega$ be a connected and simply-connected open subset of $\mathbb{R}^{3}$ and let $\left(g_{i j}\right) \in W_{\mathrm{loc}}^{1, \infty}\left(\Omega ; \mathbb{S}_{>}^{3}\right)$ be a field of positive-definite symmetric matrices.

If the Riemann curvature tensor associated with the matrix field $\left(g_{i j}\right)$ vanishes in the distributional sense, i.e., if

$$
R_{s k i j}:=g_{s \ell}\left(\partial_{i} \Gamma_{j k}^{\ell}-\partial_{j} \Gamma_{i k}^{\ell}+\Gamma_{j k}^{r} \Gamma_{i r}^{\ell}-\Gamma_{i k}^{r} \Gamma_{j r}^{\ell}\right)=0 \text { in } \mathcal{D}^{\prime}(\Omega),
$$

then there exists an immersion $\Theta \in W_{\mathrm{loc}}^{2, \infty}\left(\Omega ; \mathbb{R}^{3}\right)$ such that

$$
g_{i j}=\partial_{i} \Theta \cdot \partial_{j} \Theta \text { in } \Omega \text {. }
$$

Proof. See the proof of Theorem 4.4 in S. Mardare [9].

Our objective is to show that Theorems 3 and 4 are nothing but the "infinitesimal" versions of Theorems 5 and 6, respectively. To this end, we will show that the left-hand side of the Saint Venant equations is the linear part of the Riemann curvature tensor associated with an appropriate Riemannian metric: 
Theorem 7. Let $\Omega$ be a bounded open subset in $\mathbb{R}^{3}$ and let there be given a matrix field $\left(g_{i j}\right) \in \mathcal{C}^{2}\left(\bar{\Omega} ; \mathbb{S}_{>}^{3}\right)$ whose associated Riemann curvature tensor field vanishes in $\Omega$.

Then, for all symmetric matrix fields $\left(e_{i j}\right) \in W^{1, p}\left(\Omega ; \mathbb{S}^{3}\right), p>3$, the linear part with respect to the "increment" matrix field $\left(e_{i j}\right)$ of the covariant components of the Riemann curvature tensor associated with the metric $\left(g_{i j}+e_{i j}\right)$ are given by the relations

$$
R_{s k i j}^{\operatorname{lin}}\left(e_{i j}\right)=e_{k i \| j s}+e_{s j \| i k}-e_{k j \| i s}-e_{s i \| j k},
$$

where $e_{k i \| j s}$ denote the second-order covariant derivatives of $e_{k i}$ (cf. Section $3)$.

Proof. For all $\varepsilon>0$, we define the matrix field

$$
\left(g_{i j}(\varepsilon)\right):=\left(g_{i j}\right)+\varepsilon\left(e_{i j}\right) \in W^{1, p}\left(\Omega ; \mathbb{S}^{3}\right) .
$$

Since $W^{1, p}(\Omega) \subset \mathcal{C}^{0}(\bar{\Omega})$ by the Sobolev embedding theorem, there exists a number $\varepsilon_{0}>0$ such that, for all $0<\varepsilon<\varepsilon_{0}$, the matrix field $\left(g_{i j}(\varepsilon)\right)$ is positive definite in $\bar{\Omega}$. As in the proof Theorem 5 , this implies that $g^{k \ell}(\varepsilon) \in W^{1, p}(\Omega)$, where $\left(g^{k \ell}(\varepsilon)\right)=\left(g_{i j}(\varepsilon)\right)^{-1}$ is the inverse of the matrix field $\left(g_{i j}(\varepsilon)\right)$. Hence the Christoffel symbols

$$
\Gamma_{r j k}(\varepsilon):=\frac{1}{2}\left\{\partial_{j} g_{r k}(\varepsilon)+\partial_{k} g_{j r}(\varepsilon)-\partial_{r} g_{j k}(\varepsilon)\right\} \text { and } \Gamma_{j k}^{\ell}(\varepsilon):=g^{\ell r}(\varepsilon) \Gamma_{r j k}(\varepsilon)
$$

belong to the space $L^{p}(\Omega)$. Consequently, the Riemann curvature tensor associated with the metric $\left(g_{i j}(\varepsilon)\right)$ is well defined in the space of distributions by its mixed components

$$
R_{\cdot k i j}^{\ell}(\varepsilon):=\partial_{i} \Gamma_{j k}^{\ell}(\varepsilon)-\partial_{j} \Gamma_{i k}^{\ell}(\varepsilon)+\Gamma_{j k}^{r}(\varepsilon) \Gamma_{i r}^{\ell}(\varepsilon)-\Gamma_{i k}^{r}(\varepsilon) \Gamma_{j r}^{\ell}(\varepsilon),
$$

or by its covariant components

$$
R_{s k i j}(\varepsilon)=g_{s \ell}(\varepsilon) R_{\cdot k i j}^{\ell}(\varepsilon) .
$$

The linear part with respect to $\left(e_{i j}\right)$ of the covariant components of the Riemann curvature tensor associated with the metric $\left(g_{i j}+e_{i j}\right)$ is then defined by the limit

$$
R_{s k i j}^{\operatorname{lin}_{s}}:=\lim _{\varepsilon \rightarrow 0} \frac{R_{s k i j}(\varepsilon)}{\varepsilon} .
$$

Recall that the Riemann curvature tensor of the metric $\left(g_{i j}\right)$, whose covariant components are defined by

$$
R_{s k i j}:=g_{s \ell}\left(\partial_{i} \Gamma_{j k}^{\ell}-\partial_{j} \Gamma_{i k}^{\ell}+\Gamma_{j k}^{r} \Gamma_{i r}^{\ell}-\Gamma_{i k}^{r} \Gamma_{j r}^{\ell}\right),
$$

vanishes in $\Omega$ by assumption.

In order to compute this linear part, we expand all the above functions in power series in $\varepsilon$. Using the notation $\mathcal{O}\left(\varepsilon^{2}\right)$ for any function $f$ such that $\left(\varepsilon^{-2} f\right)$ is bounded in spaces that will be specified at each occurence, we have

$$
g_{i j}(\varepsilon)=g_{i j}+2 \varepsilon e_{i j}+\mathcal{O}\left(\varepsilon^{2}\right) \text { in } W^{1, p}(\Omega),
$$


which next implies that

$$
g^{i j}(\varepsilon)=g^{i j}-2 \varepsilon g^{i r} e_{r q} g^{q j}+\mathcal{O}\left(\varepsilon^{2}\right) \text { in } W^{1, p}(\Omega) .
$$

Consequently,

$$
\begin{aligned}
\Gamma_{k i j}(\varepsilon) & =\Gamma_{k i j}+\frac{1}{2}\left\{\partial_{i}\left(g_{k j}(\varepsilon)-g_{k j}\right)+\partial_{j}\left(g_{k i}(\varepsilon)-g_{k i}\right)-\partial_{k}\left(g_{i j}(\varepsilon)-g_{i j}\right)\right\} \\
& =\Gamma_{k i j}+\varepsilon\left(\partial_{i} e_{k j}+\partial_{j} e_{k i}-\partial_{k} e_{i j}\right)+\mathcal{O}\left(\varepsilon^{2}\right) \text { in } L^{p}(\Omega) .
\end{aligned}
$$

Likewise,

$$
\begin{aligned}
\Gamma_{i j}^{k}(\varepsilon) & =\left(g^{k \ell}-2 \varepsilon g^{k r} e_{r s} g^{s \ell}+\mathcal{O}\left(\varepsilon^{2}\right)\right)\left(\Gamma_{\ell i j}+\varepsilon\left(\partial_{i} e_{\ell j}+\partial_{j} e_{\ell i}-\partial_{\ell} e_{i j}\right)+\mathcal{O}\left(\varepsilon^{2}\right)\right) \\
& =g^{k \ell} \Gamma_{\ell i j}+\varepsilon g^{k \ell}\left(\partial_{i} e_{\ell j}+\partial_{j} e_{\ell i}-\partial_{\ell} e_{i j}\right)-2 \varepsilon g^{k r} e_{r s} g^{s \ell} \Gamma_{\ell i j}+\mathcal{O}\left(\varepsilon^{2}\right) \\
& =\Gamma_{i j}^{k}+\varepsilon g^{k \ell}\left(\partial_{i} e_{\ell j}+\partial_{j} e_{\ell i}-\partial_{\ell} e_{i j}-2 \Gamma_{i j}^{s} e_{\ell s}\right)+\mathcal{O}\left(\varepsilon^{2}\right) \\
& =\Gamma_{i j}^{k}+\varepsilon g^{k \ell}\left(e_{\ell j \| i}+e_{\ell i \| j}-e_{i j \| \ell}\right)+\mathcal{O}\left(\varepsilon^{2}\right) \text { in } L^{p}(\Omega) .
\end{aligned}
$$

Letting

$$
X_{\ell i j}:=e_{\ell j \| i}+e_{\ell i \| j}-e_{i j \| \ell} \text { and } X_{i j}^{k}=g^{k \ell} X_{\ell i j},
$$

we thus have the following relations in $L^{p}(\Omega)$ :

$$
\begin{aligned}
\Gamma_{i j}^{k}(\varepsilon) & =\Gamma_{i j}^{k}+\varepsilon X_{i j}^{k}+\mathcal{O}\left(\varepsilon^{2}\right) \\
\Gamma_{k i j}(\varepsilon) & =\Gamma_{k i j}+\varepsilon X_{k i j}+\mathcal{O}\left(\varepsilon^{2}\right) .
\end{aligned}
$$

Using these relations and the relations $R_{\cdot k i j}^{\ell}=0$ in the definition of $R_{s k i j}(\varepsilon)$, we next deduce that the relations

$$
R_{\cdot k i j}^{\ell}(\varepsilon)=\varepsilon\left(\partial_{i} X_{j k}^{\ell}-\partial_{j} X_{i k}^{\ell}+\Gamma_{j k}^{r} X_{i r}^{\ell}+X_{j k}^{r} \Gamma_{i r}^{\ell}-\Gamma_{i k}^{r} X_{j r}^{\ell}-X_{i k}^{r} \Gamma_{j r}^{\ell}\right)+\mathcal{O}\left(\varepsilon^{2}\right)
$$

hold in the space $W^{-1, p}(\Omega)$, hence also in the space $H^{-1}(\Omega)$.

Note that the covariant derivatives

$$
\begin{aligned}
X_{j k}^{\ell} \|_{i} & :=\partial_{i} X_{j k}^{\ell}-\Gamma_{i j}^{r} X_{r k}^{\ell}-\Gamma_{i k}^{r} X_{j r}^{\ell}+\Gamma_{i r}^{\ell} X_{j k}^{r}, \\
X_{\ell j k \| i} & :=\partial_{i} X_{\ell j k}-\Gamma_{i j}^{r} X_{\ell r k}-\Gamma_{i k}^{r} X_{\ell j r}-\Gamma_{i \ell}^{r} X_{r j k}, \\
g^{k \ell} \| i & :=\partial_{i} g^{k \ell}+\Gamma_{i r}^{k} g^{r \ell}+\Gamma_{i r}^{\ell} g^{k r}
\end{aligned}
$$

satisfy

$$
X_{j k}^{\ell}\left\|_{i}=g^{\ell r} X_{r j k \| i}+g^{\ell r}\right\|_{i} X_{r j k} \text { in } H^{-1}(\Omega) .
$$

Moreover, the definition of the Christoffel symbols associated with the metric $\left(g_{i j}\right)$ shows that

$$
\begin{aligned}
g^{k \ell} \|_{i} & =\partial_{i} g^{k \ell}+\frac{1}{2}\left(g^{k s} g^{r \ell}+g^{k r} g^{s \ell}\right) \Gamma_{s i r} \\
& =\partial_{i} g^{k \ell}+\frac{1}{2}\left\{g^{k s}\left(g^{r \ell} \partial_{i} g_{s r}\right)+g^{k r}\left(g^{s \ell} \partial_{i} g_{s r}\right)+\left(g^{k s} g^{r \ell}+g^{k r} g^{s \ell}\right)\left(\partial_{r} g_{i s}-\partial_{s} g_{i r}\right)\right\} \\
& =\partial_{i} g^{k \ell}-\frac{1}{2}\left\{g^{k s}\left(\partial_{i} g^{r \ell}\right) g_{s r}+g^{k r}\left(\partial_{i} g^{s \ell}\right) \partial_{i} g_{s r}\right\} \\
& =0 \text { in } L^{p}(\Omega),
\end{aligned}
$$


which, combined with the previous relations, implies that

$$
X_{j k}^{\ell} \|_{i}=g^{\ell r} X_{r j k \| i} \text { in } H^{-1}(\Omega) .
$$

Using this relation in the previous expression of $R_{\cdot k i j}^{\ell}(\varepsilon)$, we thus obtain the following relations in $H^{-1}(\Omega)$ :

$$
\begin{aligned}
R_{\cdot k i j}^{\ell}(\varepsilon) & =\varepsilon\left(X_{j k}^{\ell}\left\|_{i}-X_{i k}^{\ell}\right\|_{j}\right)+\mathcal{O}\left(\varepsilon^{2}\right) \\
& =\varepsilon g^{\ell r}\left(X_{r j k \| i}-X_{r i k \| j}\right)+\mathcal{O}\left(\varepsilon^{2}\right) \\
& =\varepsilon g^{\ell r}\left(e_{r j \| k i}-e_{j k \| r i}-e_{r i \| k j}+e_{i k \| r j}\right)+\mathcal{O}\left(\varepsilon^{2}\right)
\end{aligned}
$$

and

$$
\begin{aligned}
R_{\ell k i j}(\varepsilon) & =\varepsilon g_{\ell r} g^{r s}\left(e_{s j \| k i}-e_{j k \| s i}-e_{s i \| k j}+e_{i k \| s j}\right)+\mathcal{O}\left(\varepsilon^{2}\right) \\
& =e_{\ell j \| k i}-e_{j k \| \ell i}-e_{\ell i \| k j}+e_{i k \| \ell j}+\mathcal{O}\left(\varepsilon^{2}\right) .
\end{aligned}
$$

This completes the proof of the theorem.

Remark. The matrix field $\left(e_{i j}\right)$ was assumed in Theorem 7 to be in the space $W^{1, p}\left(\Omega ; \mathbb{S}^{3}\right)$, and not only in $L^{2}\left(\Omega ; \mathbb{S}^{3}\right)$, in order to have $\left(g_{i j}(\varepsilon)\right) \in$ $W^{1, p}\left(\Omega ; \mathbb{S}^{3}\right)$, which is the minimal regularity assumption under which the components $R_{\ell k i j}(\varepsilon)$ of the Riemannian curvature tensor are well defined in the space of distributions. However, the right hand side of the equation (13) can be extended by continuity to matrix fields $\left(e_{i j}\right)$ that belong only to the space $L^{2}\left(\Omega ; \mathbb{S}^{3}\right)$.

\section{Comparison with the Saint Venant equations in Cartesian COORDINATES}

Let $\hat{\Omega}$ be an open subset of $\mathbb{R}^{3}$. The cartesian coordinates of a point $\hat{x} \in \hat{\Omega}$ are denoted $\hat{x}_{i}$ and the partial derivative operators of the first, second, and third order of functions defined over $\hat{\Omega}$ are denoted $\hat{\partial}_{i}:=\partial / \partial \hat{x}_{i}, \hat{\partial}_{i j}:=$ $\partial^{2} / \partial \hat{x}_{i} \partial \hat{x}_{j}$, and $\hat{\partial}_{i j k}:=\partial^{3} / \partial \hat{x}_{i} \partial \hat{x}_{j} \partial \hat{x}_{k}$.

With these notations, the following theorem was proved by Ciarlet \& Ciarlet, Jr. [2].

Theorem 8. Let $\hat{\Omega}$ be a simply-connected domain of $\mathbb{R}^{3}$ and let $\left(\hat{e}_{i j}\right) \in$ $L^{2}\left(\hat{\Omega} ; \mathbb{S}^{3}\right)$ be a symmetric matrix field that satisfies the following compatibility conditions

$$
\hat{\partial}_{\ell j} \hat{e}_{i k}+\hat{\partial}_{k i} \hat{e}_{j \ell}-\hat{\partial}_{\ell i} \hat{e}_{j k}-\hat{\partial}_{k j} \hat{e}_{i \ell}=0 \text { in } H^{-2}(\hat{\Omega}) .
$$

Then there exists a vector field $\hat{\boldsymbol{v}}=\left(\hat{v}_{i}\right) \in H^{1}\left(\hat{\Omega} ; \mathbb{R}^{3}\right)$ such that

$$
\hat{e}_{i j}=\frac{1}{2}\left(\hat{\partial}_{j} \hat{v}_{i}+\hat{\partial}_{i} \hat{v}_{j}\right) \text {. }
$$


The compatibility relations in Theorem 8 are the well-known Saint Venant equations in Cartesian coordinates. Note that the Saint Venant equations in curvilinear coordinates established in Section 5 corresponds to the particular case where $\Theta=\boldsymbol{i}_{\Omega}$, which thus justifies their name. Therefore, Theorem 4 implies Theorem 8. Remarkably, the converse is also true, at least for one-to-one immersions, as we now show:

Theorem 9. Theorem 4, with the additional assumption that the immersion $\Theta: \bar{\Omega} \rightarrow \mathbb{R}^{3}$ is one-to-one, is a consequence of Theorem 8 .

Proof. As in Theorem 4, let $\Omega$ be a simply-connected domain in $\mathbb{R}^{3}$, let $\boldsymbol{\Theta} \in$ $\mathcal{C}^{3}\left(\bar{\Omega} ; \mathbb{R}^{3}\right)$ be an immersion, and let $\left(e_{i j}\right) \in L^{2}\left(\Omega ; \mathbb{S}^{3}\right)$ be a symmetric matrix field that satisfies the Saint Venant equations in curvilinear coordinates

$$
R_{s k i j}^{\operatorname{lin}}:=e_{k i \| j s}+e_{s j \| i k}-e_{k j \| i s}-e_{s i \| j k}=0
$$

in the distributional sense.

Since the mapping $\Theta$ is one-to-one, the inverse function theorem shows that $\Theta$ is in fact a $\mathcal{C}^{3}$-diffeomorphism from $\Omega$ onto $\hat{\Omega}:=\Theta(\Omega)$. The set $\hat{\Omega}$ is an open subset of $\mathbb{R}^{3}$ by the invariance of domain theorem, and it is also bounded, connected, simply-connected, with a Lipschitz-continuous boundary. This means that the set $\hat{\Omega}$ satisfies the assumptions of Theorem 8 .

Let the vector fields $\mathbf{g}_{i}$ and $\mathbf{g}^{j}$ and the Christoffel symbols $\Gamma_{i j}^{k}$ be defined as in Section 3 in terms of the immersion $\boldsymbol{\Theta}$ and let $\hat{\boldsymbol{\Theta}}=\left(\hat{\Theta}_{k}\right)$ denote the inverse mapping of the mapping $\boldsymbol{\Theta}=\left(\boldsymbol{\Theta}_{k}\right)$, i.e., $\hat{x}=\boldsymbol{\Theta}(x)$ implies $x=\hat{\boldsymbol{\Theta}}(\hat{x})$ for all $x \in \Omega$. It thus follow that

$$
\left(\hat{\partial}_{k} \hat{\Theta}_{i}\right)(\hat{x})\left(\partial_{j} \Theta_{k}\right)(x)=\delta_{j}^{i} \text { for all } x \in \Omega,
$$

or equivalently, that

$$
\boldsymbol{w}^{i}(x) \cdot \mathbf{g}_{j}(x)=\delta_{j}^{i} \text { for all } x \in \Omega,
$$

where $\boldsymbol{w}^{i}(x)$ denotes the vector in $\mathbb{R}^{3}$ whose components are $\left(\hat{\partial}_{k} \hat{\Theta}_{i}\right)(\hat{x})$. This implies that $\boldsymbol{w}^{i}(x)=\mathbf{g}^{i}(x)$ for all $x \in \Omega$, or equivalently, that

$$
\left(\hat{\partial}_{k} \hat{\Theta}_{i}\right) \circ \boldsymbol{\Theta}=\left[\mathbf{g}^{i}\right]_{k} \text { in } \Omega,
$$

where $\left[\mathbf{g}^{i}\right]_{k}$ denotes the $k$-th component of the vector field $\mathbf{g}^{i}$.

Let the matrix field $\left(\hat{e}_{p q}\right) \in L^{2}\left(\hat{\Omega} ; \mathbb{S}^{3}\right)$ be defined by

$$
\hat{e}_{p q} \circ \boldsymbol{\Theta}:=\left[\mathbf{g}^{i}\right]_{p}\left[\mathbf{g}^{j}\right]_{q} e_{i j} \text { in } L^{2}(\Omega)
$$

and let

$$
\hat{R}_{s r p q}^{\operatorname{lin}}:=\hat{\partial}_{s q} \hat{e}_{p r}+\hat{\partial}_{r p} \hat{e}_{q s}-\hat{\partial}_{s p} \hat{e}_{q r}-\hat{\partial}_{r q} \hat{e}_{p s} \text { in } H^{-2}(\hat{\Omega}) .
$$

We wish to prove that $\hat{R}_{s r p q}^{\operatorname{lin}}=0$ in $H^{-2}(\hat{\Omega})$. Noting that

$$
\partial_{i} \mathbf{g}^{k}=-\Gamma_{i j}^{k} \mathbf{g}^{j}
$$


and using the definition of the covariant derivative $e_{i j \| k}$ (see Section 3), we first obtain the relations

$$
\begin{aligned}
\left(\hat{\partial}_{r} \hat{e}_{p q}\right) \circ \boldsymbol{\Theta} & =\left[\mathbf{g}^{k}\right]_{r} \partial_{k}\left(\left[\mathbf{g}^{i}\right]_{p}\left[\mathbf{g}^{j}\right]_{q} e_{i j}\right) \\
& =\left[\mathbf{g}^{k}\right]_{r}\left[\mathbf{g}^{i}\right]_{p}\left[\mathbf{g}^{j}\right]_{q} e_{i j \| k}
\end{aligned}
$$

in $H^{-1}(\Omega)$. Using next the definition of the second-order covariant derivatives $e_{i j \| k \ell}$ (see Section 3), we obtain the relations

$$
\begin{aligned}
\left(\hat{\partial}_{s r} \hat{e}_{p q}\right) \circ \boldsymbol{\Theta} & =\left[\mathbf{g}^{\ell}\right]_{s} \partial_{\ell}\left(\left[\mathbf{g}^{k}\right]_{r}\left[\mathbf{g}^{i}\right]_{p}\left[\mathbf{g}^{j}\right]_{q} e_{i j \| k}\right) \\
& =\left[\mathbf{g}^{\ell}\right]_{s}\left[\mathbf{g}^{k}\right]_{r}\left[\mathbf{g}^{i}\right]_{p}\left[\mathbf{g}^{j}\right]_{q} e_{i j \| k \ell}
\end{aligned}
$$

in $H^{-2}(\Omega)$. Using these in the definition of $\hat{R}_{s r p q}^{\text {lin }}$, we finally obtain

$$
\begin{aligned}
\hat{R}_{s r p q}^{\operatorname{lin}} \circ \boldsymbol{\Theta} & =\left[\mathbf{g}^{\ell}\right]_{s}\left[\mathbf{g}^{k}\right]_{r}\left[\mathbf{g}^{i}\right]_{p}\left[\mathbf{g}^{j}\right]_{q}\left(e_{k i \| j \ell}+e_{\ell j \| i k}-e_{k j \| i \ell}-e_{\ell i \| j k}\right) \\
& =\left[\mathbf{g}^{\ell}\right]_{s}\left[\mathbf{g}^{k}\right]_{r}\left[\mathbf{g}^{i}\right]_{p}\left[\mathbf{g}^{j}\right]_{q} R_{\ell k i j}^{\lim }
\end{aligned}
$$

in $H^{-2}(\Omega)$, which shows that $\hat{R}_{s r p q}^{\text {lin }}=0$ in $H^{-2}(\hat{\Omega})$.

Since the assumptions of Theorem 8 are therefore satisfied, there exists a field $\hat{\boldsymbol{v}}=\left(\hat{v}_{i}\right) \in H^{1}\left(\hat{\Omega} ; \mathbb{R}^{3}\right)$ such that

$$
\hat{e}_{i j}=\frac{1}{2}\left(\hat{\partial}_{i} \hat{v}_{j}+\hat{\partial}_{j} \hat{v}_{i}\right) \text { in } L^{2}(\hat{\Omega}) .
$$

Therefore, the vector field

$$
\boldsymbol{v}:=\hat{\boldsymbol{v}} \circ \Theta
$$

belongs to the space $H^{1}\left(\Omega ; \mathbb{R}^{3}\right)$ and satisfies the relations

$$
\begin{aligned}
\frac{1}{2}\left(\partial_{i} \boldsymbol{v} \cdot \mathbf{g}_{j}+\mathbf{g}_{i} \cdot \partial_{j} \boldsymbol{v}\right) & =\partial_{i} \Theta_{p} \partial_{j} \Theta_{q} \frac{\left(\hat{\partial}_{p} \hat{\boldsymbol{v}} \cdot \hat{\partial}_{q} \boldsymbol{i}_{\hat{\Omega}}+\hat{\partial}_{q} \hat{\boldsymbol{v}} \cdot \hat{\partial}_{p} \boldsymbol{i}_{\hat{\Omega}}\right) \circ \boldsymbol{\Theta}}{2} \\
& =\left[\mathbf{g}_{i}\right]^{p}\left[\mathbf{g}_{j}\right]^{q} \frac{\left(\hat{\partial}_{p} \hat{v}_{q}+\hat{\partial}_{q} \hat{v}_{p}\right) \circ \boldsymbol{\Theta}}{2} \\
& =\left[\mathbf{g}_{i}\right]^{p}\left[\mathbf{g}_{j}\right]^{q}\left(\hat{e}_{p q} \circ \boldsymbol{\Theta}\right) \text { in } L^{2}(\Omega),
\end{aligned}
$$

where $\left[\mathbf{g}_{i}\right]^{p}$ denotes the $p$-th component of the vector field $\mathbf{g}_{i}$. Then the definition of the functions $\hat{e}_{p q}$ shows that

$$
\frac{1}{2}\left(\partial_{i} \boldsymbol{v} \cdot \mathbf{g}_{j}+\mathbf{g}_{i} \cdot \partial_{j} \boldsymbol{v}\right)=\left[\mathbf{g}_{i}\right]^{p}\left[\mathbf{g}_{j}\right]^{q}\left[\mathbf{g}^{k}\right]_{p}\left[\mathbf{g}^{\ell}\right]_{q} e_{k \ell}=e_{i j}
$$

in $L^{2}(\Omega)$. This completes the proof.

Remark. If $\Theta \in \mathcal{C}^{3}\left(\bar{\Omega} ; \mathbb{R}^{3}\right)$ is an immersion, but not necessarily one-to-one, then the inverse function theorem shows that the mapping $\boldsymbol{\Theta}$ is locally oneto-one, that is, for all $x \in \bar{\Omega}$, there exists an open ball $B\left(x, r_{x}\right)$ with $r_{x}>0$ such that the set $\Omega_{x}:=\Omega \cap B\left(x, r_{x}\right)$ is simply-connected and the mapping $\left.\Theta\right|_{\bar{\Omega}_{x}}: \bar{\Omega}_{x} \rightarrow \mathbb{R}^{3}$ is one-to-one. 
Since the set $\bar{\Omega}$ is compact, there exists a finite covering of $\Omega$ with simplyconnected domains $\Omega_{n}, 1 \leq n \leq N$, such that the mappings

$$
\Theta_{n}:=\left.\Theta\right|_{\bar{\Omega}_{n}}: \bar{\Omega}_{n} \rightarrow \mathbb{R}^{3}
$$

are one-to-one.

Let $\left(e_{i j}\right) \in L^{2}\left(\Omega ; \mathbb{S}^{3}\right)$ be a matrix field that satisfies the Saint Venant equations in curvilinear coordinates and let $e_{i j}^{n}:=e_{i j_{\Omega_{n}}}$. Then Theorem 9 shows that, for all $n \in\{1,2, \ldots, N\}$, there exists a vector field $\boldsymbol{v}^{n} \in H^{1}\left(\Omega_{n} ; \mathbb{R}^{3}\right)$ such that

$$
e_{i j}^{n}=\varepsilon_{i j}\left(\boldsymbol{v}^{n}\right):=\frac{1}{2}\left(\partial_{i} \boldsymbol{v}^{n} \cdot \mathbf{g}_{j}+\mathbf{g}_{i} \cdot \partial_{j} \boldsymbol{v}^{n}\right) \text { in } L^{2}\left(\Omega_{n} ; \mathbb{S}^{3}\right) .
$$

On the other hand, the uniqueness result furnished by Theorem 3 of Ciarlet \& C. Mardare [3] (see also the Remark at the end of Section 6) shows that if two vector fields $\boldsymbol{u}, \boldsymbol{v} \in H^{1}\left(U ; \mathbb{R}^{3}\right)$ satisfy $\varepsilon_{i j}(\boldsymbol{u})=\varepsilon_{i j}(\boldsymbol{v})$ in a connected open set $U$, then there exist vectors $\mathbf{a}, \mathbf{b} \in \mathbb{R}^{3}$ such that

$$
\boldsymbol{v}(x)=\boldsymbol{u}(x)+(\mathbf{a}+\mathbf{b} \wedge \boldsymbol{\Theta}(x)) \text { for almost all } x \in U .
$$

If the open set $\Omega$ is connected, these two results allow to establish Theorem 4 as a consequence of Theorem 8 without assuming that the immersion $\Theta: \Omega \rightarrow \mathbb{R}^{3}$ is one-to-one by following the proof of Theorem 7 from S. Mardare [8].

Acknowledgements. The work described in this paper was substantially supported by a grant from the Research Grants Council of the Hong Kong Special Administrative Region, China [Project No. 9041076, CityU 100105].

\section{REFERENCES}

[1] Adams, R.A.: Sobolev Spaces, Academic Press, New York, 1975.

[2] Ciarlet, P.G. and Ciarlet, P. Jr.: Another approach to linearized elasticity and a new proof of Korn's inequality, Math. Models Methods Appl. Sci. 15 (2005), 259-271.

[3] Ciarlet, P.G. and Mardare, C.: On rigid and infinitesimal rigid displacements in three-dimensional elasticity, Mathematical Models and Methods in Applied Sciences 13 (2003), 1589-1598.

[4] Ciarlet, P.G. and Mardare, C.: Continuity of a deformation in $H^{1}$ as a function of its Cauchy-Green tensor in $L^{1}$, J. of Nonlinear Science 14 (2004), 415-427.

[5] Ciarlet, P.G., Gratie, L., Mardare, C., and Shen, M.: Saint Venant equations on a surface, to appear

[6] Grisvard P.: Elliptic Problems in Nonsmooth Domains, Pitman, Boston, 1985.

[7] Nečas, J.: Les Méthodes Directes en Théorie des Equations Elliptiques, Masson, Paris, 1967.

[8] Mardare, S.: On Pfaff systems with $L^{p}$ coefficients and their applications in differential geometry, J. Math. Pures Appl. 84 (2005), 1659-1692.

[9] Mardare, S.: On isometric immersions of a Riemannian space with little regularity, Analysis and Applications 2 (2004), 193-226.

[10] Mardare, S.: Sur quelques problèmes de géomètrie différentielle liés à la théorie de l'élasticité, Doctoral Disertation, Université Paris 6, 2003. 
Philippe G. Ciarlet, Department of Mathematics, City University of Hong Kong, 83 Tat Chee Avenue, Kowloon, Hong Kong, E-mail address: mapgc@cityu.edu.hk

Cristinel Mardare, Université Pierre et Marie Curie-Paris6, UMR 7598 Laboratoire Jacques-Louis Lions, Paris, F-75005 France, E-mail address: mardare@ann.jussieu.fr

Ming Shen, Department of Mathematics, City University of Hong Kong, 83 Tat Chee Avenue, Kowloon, Hong Kong, E-mail address: geoffrey.shen@student.cityu.edu.hk 